\title{
Genetic variations in the osteopontin promoters T-443C and G-I56GG increase carotid intima-media thickness
}

This article was published in the following Dove Press journal:

International Journal of General Medicine

12 May 2016

Number of times this article has been viewed

\section{Yuyun Yueniwati' \\ Valentina Yurina ${ }^{2}$ \\ Nurus Sobah ${ }^{2}$ \\ Endang Rahayu ${ }^{2}$}

'Radiology Department, ${ }^{2}$ Clincal Pharmacy Department, Pharmacy Study Program, Faculty of Medicine, Brawijaya University, Malang, Indonesia

Correspondence: Yuyun Yueniwati Radiology Department, Faculty of Medicine, Brawijaya University, JI Veteran Malang, Malang 65I45, Indonesia

Tel +62341569 II7

Fax +62 34I 564755

Email yuyun@ub.ac.id

\begin{abstract}
Carotid intima-media thickness (CIMT) is a clear predictor of atherosclerosis. The increase of CIMT is affected by mutations in the osteopontin (OPN) promoters. The purpose of this study was to examine genetic variations in OPN promoters T-443C and G-156GG, identified in Javanese children with ischemic stroke parents, and to investigate their relationship with the increase of CIMT. A case-control analytic study was performed on 20 case and 12 control samples. Case samples were Javanese children aged between 10 to 21 years with ischemic stroke parents. Control samples were children with healthy parents. Mutations of T-443C and G-156GG were determined by employing polymerase chain reaction. Results of sequencing were analyzed using CLC Main Workbench 6.0. CIMT was defined using ultrasound. Genetic variations of T-443C were identified in six samples. Likewise, genetic variations of G-156GG were identified in six samples. Genetic variations in the OPN promoters T-443C and G-156GG were not potential risk factors in an increase of CIMT ( $P=0.654$ and $P=0.654)$. This study proves that genetic variations could be identified at the points of T-443C and G-156GG in children with ischemic stroke parents. Although statistically insignificant, the tendency to increase CIMT occurs in children with genetic variations. Children with ischemic stroke parents have thicker CIMT than children of healthy parents.
\end{abstract}

Keywords: carotid intima-media thickness, genetic variation, ischemic stroke, osteopontin promoters

\section{Introduction}

Despite the decrease in stroke mortality rate in the mid-20th century, the incidence of stroke remains high and is the third major cause of death in the world. Around 150,000 people die and more than 700,000 people suffer from stroke every year. ${ }^{1}$ The prevalence of stroke in Indonesia is $8.3 \%$ per 1,000 population, whereas $6 \%$ per 1,000 population is diagnosed with stroke. This indicates that $72.3 \%$ of stroke cases in Indonesia have been diagnosed and $19.1 \%$ of them are among the Javanese population. ${ }^{2}$

Based on its mechanism of occurrence, stroke is divided into two categories, namely, ischemic and hemorrhagic stroke. ${ }^{3}$ The occurrence of ischemic stroke is more frequent than hemorrhagic stroke. ${ }^{3}$ Ischemic stroke is a condition associated with atherosclerosis, which is a vascular abnormality due to formation of plaques in the arteries and causes hardening and constriction of blood vessels resulting in decreased blood supply to the heart and other organs. ${ }^{3,4}$ Atherosclerosis can lead to heart attack, stroke, and even death. Early sign of atherosclerosis is the increase of carotid intima-media thickness (CIMT). ${ }^{4,5}$ submit your manuscript | www.dovepress.con Dovepress http://dx.doi.org// 0.2147/IJGM.S94537 
A study conducted by Brenner et $\mathrm{al}^{6}$ revealed that genetic factors can cause an increase in CIMT. The study suggested that osteopontin (OPN) is closely related to the increase of CIMT. OPN is a phosphoglycoprotein adhesive that indicates some functions in different physiological and pathological processes including bone repair, cell-mediated immunity, protection and reestablishment of tissue integrity during inflammatory processes, and metastases. ${ }^{7,8}$ Polymorphisms in OPN promoters can occur in various regions, such as T-443C, T-66G, and G-156GG. Although backgrounds of these mutations are varied, most of them show the mutation effects toward atherosclerosis progression. ${ }^{9,10}$ The presence of genetic variations in the OPN promoters with zinc finger motif leads to the occurrence of sticking MYT1 transcription factor. This process causes affinity binding of RNA polymerase toward the promoter to increase. Consequently, OPN overexpression occurs, which results in an earlier increase of CIMT with faster progression. ${ }^{9,11}$ This study aimed to analyze the genetic variations in gene coding for OPN (SNP T-443C and G-156GG) among Javanese Indonesian children with ischemic stroke parents and its relationship with the increase of CIMT. Up to now, there have been limited studies on polymorphism in Indonesia, especially related to atherosclerosis. This study will give more information about polymorphism related with atherosclerosis in Indonesia. Furthermore, the findings of this study will give a deeper understanding about the influence of OPN polymorphism toward atherosclerosis progression; therefore, precautions can be started earlier.

\section{Materials and methods}

\section{Subjects and blood collection}

The case-control study was designed using analytic observational approach. Research participants were Javanese Indonesian children aged between 10 to 21 years, who were divided into two groups, that is, case and control groups. The case group consisted of 20 children with biological parents (father and/or mother) who were ischemic stroke patients. The parents were or had been hospitalized, or being or had been treated at the Neurology Clinic of Saiful Anwar General Hospital, Malang, Indonesia. The control group consisted of 12 children with healthy biological parents of similar health background (40-50 years old, nonsmokers, not obese). Participants and parents provided written informed consent together; parents wrote the informed consent for participants under 15 years old. Blood samples were taken after the participants fasted for 12 hours.
This study was approved by the Ethics Committee of Faculty of Medicine, Brawijaya University, Malang, Indonesia.

\section{Determining CIMT using high-resolution ultrasonography}

CIMT measurements were conducted in the Radiology Department of Saiful Anwar General Hospital. CIMT measurements for all samples were performed using ultrasound Logic S6 (GE Healthcare Bio-Sciences Corp., Piscataway, NJ, USA). CIMT was measured from the base to the carotid bulb, in three angles, that is, transversal, posterolateral, and anterolateral. ${ }^{12}$

\section{Analysis on genetic variants}

Blood samples of case and control groups were taken and collected in tubes containing ethylenediaminetetraacetic acid. DNA chromosomes were then isolated using the procedure of the extraction kit Wizard ${ }^{\circledR}$ Genomic DNA purification kit (Promega Corporation, Fitchburg, WI, USA). OPN promoter regions were amplified using OPN-specific primers in the form of forward primer OPN1 5 'ATTACAATTCGTGACTGCCTGCC- $3^{\prime}$ and reverse primer OP2 with the base sequence of $5^{\prime}$-TGTACCTTGGTCGGCGTTTG- $3{ }^{\prime} .{ }^{10}$ Polymerase chain reaction conditions were as follows: predenaturation at $94^{\circ} \mathrm{C}$ for 2 minutes, denaturation at $94^{\circ} \mathrm{C}$ for 20 seconds, annealing at $54^{\circ} \mathrm{C}$ for 10 seconds, elongation at $72^{\circ} \mathrm{C}$ for 45 seconds, postelongation at $72^{\circ} \mathrm{C}$ for 5 seconds, and final hold at $4{ }^{\circ} \mathrm{C}$ for 10 minutes with 35 repeated cycles. The results were analyzed using the polymerase chain reaction product sequencing program (CLC Main Workbench, CLC Bio, Qiagen, Denmark) to determine the location of the mutations.

\section{Statistical analysis}

Results of the genetic variation analysis were processed using Fisher's exact test.

\section{Results}

\section{Analysis of genetic variations T-443C and G-I56GG}

Results of two-way direction of sequencing with forward and reverse primers indicated mutations in the same six samples, that is, substitution of thymine base -443 with cytosine. Mutations occurred in four samples of the case group and two samples of the control group. The results of analysis on mutations in base number -443 using CLC Main Workbench 6.0 program are presented in Figure 1.

In the meantime, results of the sequencing analysis on base number -156 showed six occurrences of mutations 


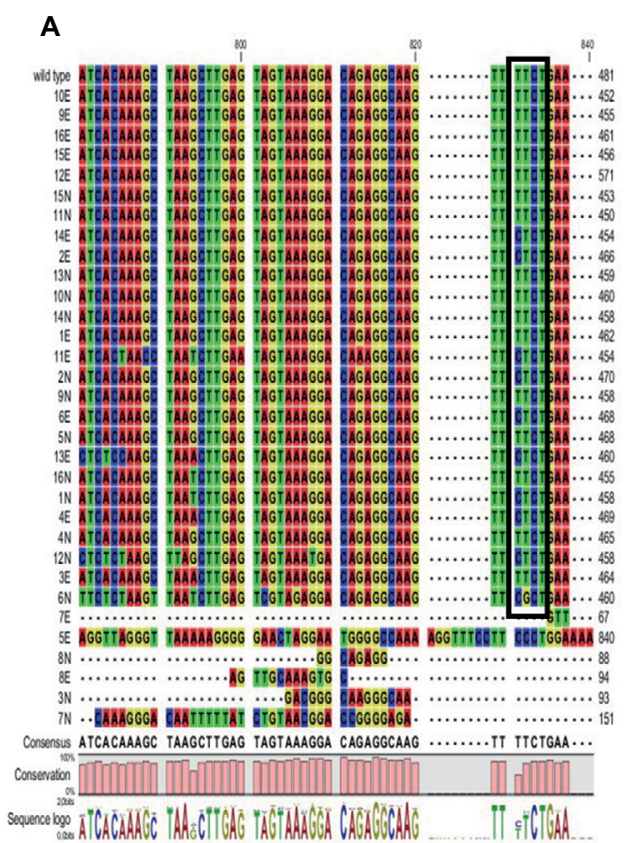

B

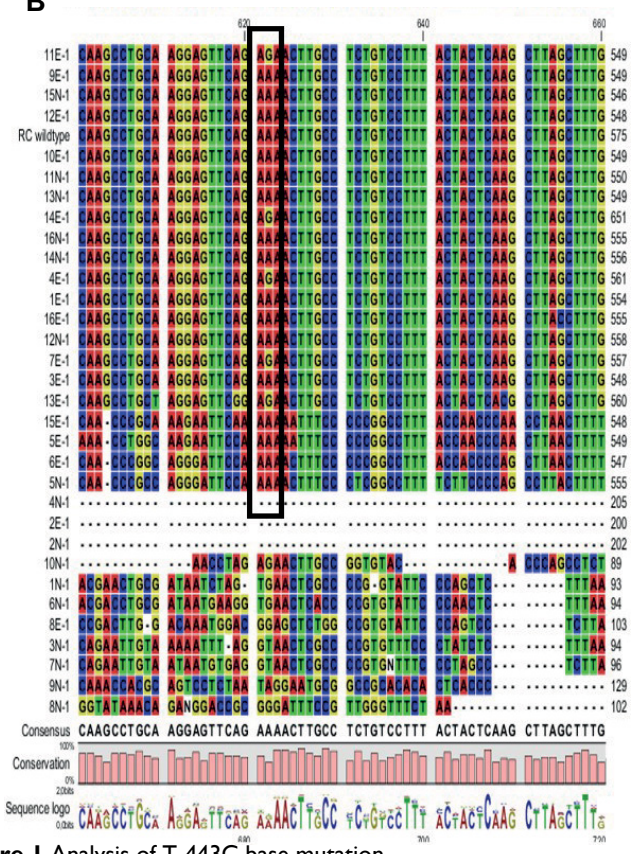

Figure I Analysis of T-443C base mutation.

Notes: (A) Results of sequencing performed with OPN forward primer; (B) results of sequencing performed with OPN reverse primer.

Abbreviation: OPN, osteopontin.

of guanine base insertion, that is, in two case samples and four control samples. The mutations were confirmed using two-way sequencing with forward and reverse primers. The results of analysis on mutations in base number -156 using CLC Main Workbench 6.0 program are given in Figure 2.

Mutations of -443 and -156 occurred in different subjects. None of the subjects with two mutations were analyzed simultaneously. Data on T-443C and G-156GG mutations, along with CIMT thickness of each subject, are presented in Table 1.
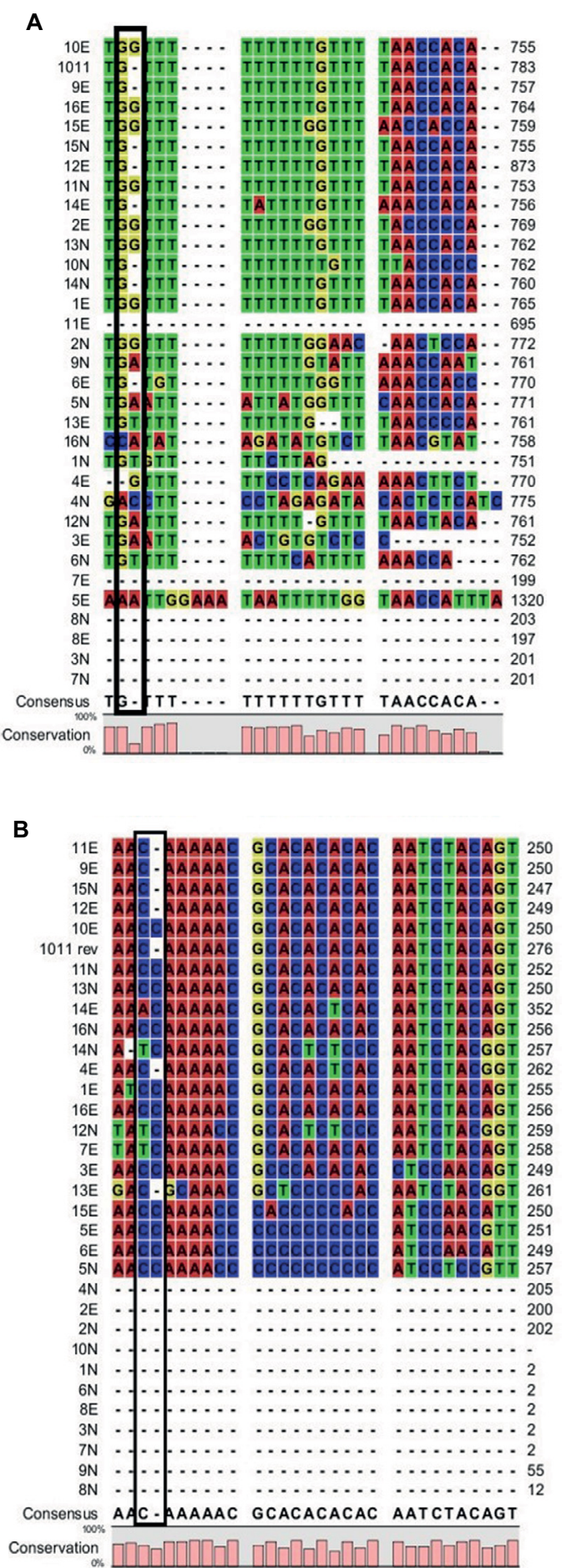

Figure 2 Analysis of G-I56GG base mutation.

Notes: (A) Results of sequencing performed with OPN forward primer; (B) results of sequencing performed with OPN reverse primer.

Abbreviation: OPN, osteopontin.

\section{Significant relation test between mutation group and nonmutation group toward CIMT}

Analysis of the relations between mutations and CIMT was done by employing hypothesis testing, that is, by calculating the risks of genetic variations toward CIMT. The result 
Table I Data of T-443C and G-I56GG mutations and CIMT

\begin{tabular}{llll}
\hline $\begin{array}{l}\text { Subjects with } \\
\text { T-443C mutation }\end{array}$ & CIMT (mm) & $\begin{array}{l}\text { Subjects with } \\
\text { G-I56GG mutation }\end{array}$ & $\begin{array}{l}\text { CIMT } \\
\mathbf{( m m )}\end{array}$ \\
\hline IIE (S) & 0.60 & I6E (S) & 0.50 \\
I4E (S) & 0.60 & IIN (S) & 0.55 \\
$4 E(S)$ & 0.60 & I SN (C) & 0.60 \\
6N (S) & 0.50 & I5E (C) & 0.45 \\
I3E (C) & 0.40 & IE (C) & 0.50 \\
IN (C) & 0.45 & IOE (C) & 0.40 \\
\hline
\end{tabular}

Note: Testing significant relationship between mutation group and nonmutation group toward CIMT.

Abbreviations: C, control; CIMT, carotid intima-media thickness; S, sample.

Table 2 Relationship between mutations and CIMT

\begin{tabular}{lll}
\hline Variable & $\begin{array}{l}\boldsymbol{P} \text { (Fisher's } \\
\text { exact test) }\end{array}$ & OR (95\% CI) \\
\hline Total mutation-CIMT & 0.273 & $3.00(0.67 \mathrm{I}-13.404)$ \\
T-443C mutation-CIMT & 0.654 & $2.33(0.362-15.053)$ \\
G-I56GG mutation-CIMT & 0.654 & $2.33(0.362-15.053)$ \\
\hline
\end{tabular}

Abbreviations: $\mathrm{Cl}$, confidence interval; $\mathrm{CIMT}$, carotid intima-media thickness; $\mathrm{OR}$, odds ratio.

was processed using Fisher's exact test (Table 2). This test revealed that there was no significant relationship between genetic mutations with CIMT $(P=0.273)$. The $P$-value of genetic variation G-156GG with CIMT was 0.654 and the $P$-value of T-143C mutation with CIMT was 0.654 , which means that genetic variation was not a significant risk factor in the increase of CIMT.

\section{Analysis of CIMT mean in case and control subjects}

Analysis using independent $t$-tests showed that the cases had significantly higher mean values of both right and left CIMT (Table 3).

\section{Discussion}

OPN is a protein that has a role in the pathogenesis of certain diseases such as cancer, atherosclerosis, and chronic inflammatory diseases. ${ }^{13,14} \mathrm{OPN}$ is expressed in smooth muscle cells, angiogenic endothelial cells, and macrophages on the lesions of atherosclerosis. OPN modulates proliferation, migration, and accumulation of smooth muscle and endothelial cells, which are involved in the process of vascular repair and remodeling. In addition, OPN is also suggested to have a role in deposition of minerals in conditions such as arterial injuries and vascular diseases, because OPN has an antihardening (anticalcification) effect on the blood vessels and is capable of enhancing mineral reposition. ${ }^{15}$ This suggests that the expression of OPN in atherosclerosis plaque can be a marker of the severity of atherosclerosis as well as
Table 3 CIMT mean (SD) of cases and controls

\begin{tabular}{llll}
\hline CIMT & Case $(\mathbf{m m})$ & Control $(\mathbf{m m})$ & P-value \\
\hline Right CIMT mean & $0.61(0.015)$ & $0.53(0.016)$ & 0.032 \\
Left CIMT mean & $0.60(0.013)$ & $0.51(0.018)$ & 0.034 \\
Right-left CIMT mean & $0.61(0.012)$ & $0.52(0.015)$ & 0.021 \\
\hline
\end{tabular}

Abbreviations: CIMT, carotid intima-media thickness; SD, standard deviation.

blood vessel hardening (calcification). High concentrations of osteoprotegerin and OPN indicate the potential atherosclerosis plaque to be unstable plaque. Both proteins are associated with artery decalcification, a process that leads to the occurrence of atherosclerosis plaque rupture. ${ }^{8,9}$

\section{Influence of genetic variations in the OPN promoter T-443C on CIMT}

A genetic variation in the OPN promoter T-443C is one of the genetic factors suspected to have relation with CIMT. The -443 base is a promoter site which is a part of DNA in which transcription starts. The transcription occurs when sequences of promoters bind to RNA polymerase II. Genetic variation of T-443C in the promoter sequences leads to OPN overexpression. ${ }^{9}, 16$ In the same study, Giacopelli et al ${ }^{9}$ reported that transcription factors, which are bound to OPN sequence promoter, were MYT1 with zinc finger motifs. The zinc finger is one of the motifs mediating regulatory protein binding with DNA. Thus, the regulatory proteins can specifically bind with high affinity, so that they can increase the activity of transcription. ${ }^{9}$ As for the genetic variation T-443C, it was identified that there were six samples undergoing mutations - four samples from the case group and two samples from the control group. Four out of six samples undergoing genetic variations were from the case group subjects who displayed thicker CIMT than the two samples from the control group undergoing genetic variations. Based on CIMT value, it can be stated that children with parents who had ischemic stroke history were identified to have thicker CIMT.

This result is in accordance with previous research results, which mentioned that genetic variations in the OPN promoter T-443 had significant effect in the increase of CIMT due to disorders in the transcription activity of OPN gene. ${ }^{6,9}$ The genetic variation $\mathrm{T}-443 \mathrm{C}$ in the OPN promoter site results in the occurrence of binding transcription factors of MYT1 with zinc finger motifs in the promoter site.,

The binding transcription factors increase the affinity of polymerase RNA toward promoter, which also causes an increase in transcription activity. As a result, OPN overexpression will lead to earlier and faster vascular smooth muscle proliferation. Hence, narrowing and hardening blood vessels 
also occur more quickly, which then leads to decreased blood supply to various organs, particularly to the brain. The decline in the blood supply containing oxygen to the brain causes ischemic stroke. ${ }^{8,9}$

Two other samples experiencing genetic variation unexpectedly came from the control group subjects who were expected not to undergo genetic variations. This was possibly due to other conditions marked by the occurrence of genetic variations in the OPN promoter T-443C, such as history of myocardial infarction in parents, obesity, and cancer. ${ }^{17,18}$ Some previous research results ${ }^{19,20}$ also described that genetic variations in the OPN promoter $\mathrm{T}-443 \mathrm{C}$ resulting in plasma OPN overexpression were not only always associated with increase in CIMT, but also with some other diseases, such as systemic lupus erythematous, multiple sclerosis, urolithiasis, and primary biliary cirrhosis. Likewise, OPN overexpression was identified in several other conditions such as chronic arthritis, myocardial infarction, interstitial fibrosis of kidney due to obstructive uropathy, and in the process of injury healing which is one of the physiological functions of OPN. Besides, OPN overexpression could also be a predictor of atherosclerosis risk in patients with essential hypertension. The results of previous research indicated that genetic variations in OPN promoter T-443C identified in the control group were possibly associated with the conditions above. This explains why the control group experiencing genetic variations in the OPN promoter T-443 did not undergo an increase in CIMT. ${ }^{21}$

\section{The influence of genetic variations in the OPN promoter G-I56GG toward CIMT}

The study results showed that there were two sample cases experiencing an increase in CIMT. This reinforces the results of previous studies which identified a tendency of genetic variation at point $\mathrm{G}-156 \mathrm{GG}$ to be associated with increased CIMT. ${ }^{6,21}$ One of the studies explained that the presence of two guanine bases in the DNA sequence at the site of -156 caused the formation of binding sites for RUNT factor. ${ }^{9}$ RUNX2 is a transcription factor which can bind to the binding site formed through the insertion of $\mathrm{G}$ in the base sequence. The bond has a great affinity and, thus, increases the transcriptional activity of OPN promoter. The OPN promoter transcriptional activity causes an increase in the OPN concentration of plasma. OPN is widely expressed in macrophages in human adipose tissues, whose amount is also associated with obesity. An increase in the transcriptional activity of OPN also results in an increase in the local recruitment of monocytes at the site of injury in the arterial wall, thereby increasing the risk of atherosclerosis. ${ }^{13,22}$

\section{Conclusion}

In conclusion, genetic variations in the OPN promoters T-443C and G-156GG were found in the population of Javanese children with ischemic stroke parents. Although statistically insignificant, the existence of one of these mutations resulted in the tendency to increase CIMT. The children with ischemic stroke parents have thicker carotid intima-media than those with normal parents.

\section{Acknowledgment}

This work was supported by grants from the Faculty of Medicine, Brawijaya University, Malang, Indonesia.

\section{Disclosure}

The authors report no conflicts of interest in this work.

\section{References}

1. Mozaffarian D, Benjamin EJ, Go AS, et al. Heart disease and stroke statistics - 2015 update: A report from the American Heart Association. Circ. 2015;131 (4):e29-322.

2. Kusuma Y, Venketasubramanian N, Kiemas LS, Misbach J. Burden of stroke in Indonesia. Int J Stroke. 2009;4(5):379-380.

3. Sacco RL, Kasner SE, Broderick JP, et al. An updated definition of stroke for the 21st century: a statement for healthcare professionals from the American Heart Association/American Stroke Association. Stroke. 2013;44(7):2064-2089.

4. Sahoo R, Krishna MV, Subrahmaniyan DKS, Dutta TK, Elangovan S. Common carotid intima-media thickness in acute ischemic stroke: A case control study. Neurol India. 2009;57(5):627-630.

5. Barra S, Gaeta G, Cuomo S, et al. Early increase of carotid intima-media thickness in children with parental history of premature myocardial infarction. Heart. 2009;95(8):642-645.

6. Brenner D, Labreuche J, Touboul P-J, et al. Cytokine polymorphisms associated with carotid intima-media thickness in stroke patients. Stroke. 2006;37(7):1691-1696.

7. Sase SP, Ganu J V, Nagane N. Osteopontin: A novel protein molecule. Ind Med Gaz. 2012;62-66.

8. Isoda K. Osteopontin plays an important role in the development of medial thickening and neointimal formation. Circ Res. 2002;91(1):77-82.

9. Giacopelli F, Marciano R, Pistorio A, et al. Polymorphisms in the osteopontin promoter affect its transcriptional activity. Physiol Genomics. 2004;20(1):87-96.

10. Lusis AJ, Fogelman AM, Fonarow GC. Genetic basis of atherosclerosis: part I: new genes and pathways. Circulation. 2004;110(13):1868-1873.

11. Lusis AJ, Fogelman AM, Fonarow GC. Genetic basis of atherosclerosis: part II: clinical implications. Circulation. 2004;110(14):2066-2071.

12. Casella IB, Presti C, Porta RMP, Sabbag CRD, Bosch MA, Yamazaki Y. A practical protocol to measure common carotid artery intima-media thickness. Clinics. 2008;63(4):515-520.

13. Scatena M, Liaw L, Giachelli CM. Osteopontin: a multifunctional molecule regulating chronic inflammation and vascular disease. Arterioscler Thromb Vasc Biol. 2007;27(11):2302-2309.

14. Lee T-Y, Lin J-T, Wu C-C, et al. Osteopontin promoter polymorphisms are associated with susceptibility to gastric cancer. J Clin Gastroenterol. 2013;47(6):55-59.

15. Mazzali M, Kipari T, Ophascharoensuk V, Wesson JA, Johnson R, Hughes J. Osteopontin--a molecule for all seasons. QJM. 2002;95(1):3-13. 
16. Mendioroz M, Fernández-Cadenas I, Rosell A, et al. Osteopontin predicts long-term functional outcome among ischemic stroke patients. J Neurol. 2011;258(3):486-493.

17. Pan H-W, Ou Y-H, Peng S-Y, et al. Overexpression of osteopontin is associated with intrahepatic metastasis, early recurrence, and poorer prognosis of surgically resected hepatocellular carcinoma. Cancer. 2003;98(1):119-127.

18. Zhao F, Chen X, Meng T, Hao B, Zhang Z, Zhang G. Genetic polymorphisms in the osteopontin promoter increases the risk of distance metastasis and death in Chinese patients with gastric cancer. $B M C$ Cancer. 2012;12(1):477.
19. Kaleta B. Role of osteopontin in systemic lupus erythematosus. Arch Immunol Ther Exp (Warsz). 2014;475-482.

20. Yueniwati Y, Yurina V, Indra MR. Thicker Carotid Intima Media thickness in children with monocyte chemoattractant protein-1: A-2138T and A-2464G mutation. Neurol Res Int. 2014;1-6.

21. Fuentes L De, Gu CC, Mathews S, et al. Osteopontin promoter polymorphism is associated with increased carotid intima-media thickness. J Am Soc Echocardiogr. 2009;21(8):954-960.

22. Kurata M, Okura T, Watanabe S, Fukuoka T, Higaki J. Osteopontin and carotid atherosclerosis in patients with essential hypertension. Clin Sci (Lond). 2006;111(5):319-324.

\section{Publish your work in this journal}

The International Journal of General Medicine is an international, peer-reviewed open-access journal that focuses on general and internal medicine, pathogenesis, epidemiology, diagnosis, monitoring and treatment protocols. The journal is characterized by the rapid reporting of reviews, original research and clinical studies across all disease areas.
The manuscript management system is completely online and includes a very quick and fair peer-review system, which is all easy to use. Visit http://www.dovepress.com/testimonials.php to read real quotes from published authors.

Submit your manuscript here: https://www.dovepress.com/international-journal-of-general-medicine-journal 